\title{
Selective advantage for conservative viruses
}

\section{Citation}

Brumer, Yisroel, and Eugene I. Shakhnovich. 2005. "Selective Advantage for Conservative Viruses." Physical Review E71 (3): 031903. https://doi.org/10.1103/PhysRevE.71.031903.

\section{Permanent link}

http://nrs.harvard.edu/urn-3:HUL.InstRepos:41534504

\section{Terms of Use}

This article was downloaded from Harvard University's DASH repository, and is made available under the terms and conditions applicable to Other Posted Material, as set forth at http:// nrs.harvard.edu/urn-3:HUL.InstRepos:dash.current.terms-of-use\#LAA

\section{Share Your Story}

The Harvard community has made this article openly available.

Please share how this access benefits you. Submit a story.

Accessibility 


\title{
A Selective Advantage for Conservative Viruses
}

\author{
Yisroel Brumer and Eugene I. Shakhnovich \\ Harvard University, 12 Oxford Street, Cambridge, Massachusetts 02138
}

(Dated: November 14, 2018)

\begin{abstract}
In this letter we study the full semi-conservative treatment of a model for the co-evolution of a virus and an adaptive immune system. Regions of viability are calculated for both conservatively and semiconservatively replicating viruses interacting with a realistic semi-conservatively replicating immune system. The conservative virus is found to have a selective advantage in the form of an ability to survive in regions with a wider range of mutation rates than its semi-conservative counterpart. This may help explain the existence of a rich range of viruses with conservatively replicating genomes, a trait which is found nowhere else in nature.

PACS numbers: $87.14 \mathrm{Gg}, 87.23 \mathrm{Kg}, 87.10+\mathrm{e}$
\end{abstract}

DNA is often called the molecule of life. The vast majority of organisms in nature store their genetic information in the form of double stranded DNA, which provides a number of benefits over its close relative and likely predecessor, $\mathrm{RNA}^{1}$. These benefits include a predictable secondary structure and a resistance to auto-catalytic cleavage and hence a longer half-life. However, viruses stand out as a notable exception to the "DNA world", employing a variety of methods for genetic storage including single stranded RNA (e.g. tobacco mosaic virus), double stranded RNA (such as reovirus), linear and circular single stranded DNA (including parvovirus and bacteriophage $\phi \mathrm{X} 174$, respectively) and a variety of double stranded DNA types (examples of which include bacteriophage $\mathrm{T} 4$, polyoma virus and poxvirus). While double stranded DNA replicates semi-conservatively, many of these viruses replicate conservatively, wherein multiple copies of a single strand are made, and the original strand is conserved. This suggests that either the benefits of DNA must be less for viral species (possibly due to a shorter genome length or unusual life cycles) and/or a selective advantage must exist for conservatively replicating viruses. In this paper we use the the quasispecies model to search for such an advantage.

Introduced by Eigen in 1971, the quasispecies mode ${ }^{2,3}$ has been used to study various characteristics of conservatively replicating organisms ranging from equilibrium data to punctuated evolution $4,5,6,7,8,9,10,11,12,13,14,15$. The model consists of a population of independently replicating genomes $\phi$, each of which is made up of a set of "letters" $s_{1} s_{2} \cdots s_{n}$ chosen from an "alphabet" of size S. $\mathrm{S}$ is usually chosen to be two for simplicity or four, as in this paper, to model the nucleotides A, C, T and G. Each possible genome is assigned a fitness that dictates its fecundity. This mapping of fitness to genotype can be represented by a unique "fitness landscape". The process of replication includes a probability of point mutation per base pair $\epsilon$ that is generally assumed to be genome-independent. By associating phenotype with genotype and assuming a first-order dependence of the growth rate on concentration, a set of differential equations can be solved to describe the competition between various genotypes ${ }^{2,3}$. Although the model incorporates numerous approximations, it is well suited to describing small RNA genomes and viruses and many of its predictions have been experimentally verified. One of the major successes of the model lies in recent work on novel anti-viral therapies 16.17 .

The quasispecies model has recently been extended to the coevolution of hosts and parasites and the particular case of an adaptive immune system interacting with a virus ${ }^{18,19}$. Viruses make detrimental use of host biochemical processes while the immune system expends enormous effort to keep viral concentrations as low as possible. As the immune system develops new defenses, the virus must adapt to defeat them. The immune system must then evolve to destroy the newly resistant strains, and a non-linear co-evolving feedback loop is created. To model this behavior, the immune system and virus are both assumed to evolve on a single fitness peak landscape, where the fitness of all genomes are equal with the sole exception of a single master sequence of far greater fitness, or

$$
A(\phi)=\left\{\begin{array}{cc}
\eta & \phi \neq \phi_{0} \\
\sigma \gg \eta & \phi=\phi_{0}
\end{array},\right.
$$

where $A(\phi)$ represents the fitness of genome $\phi$ and $\{\sigma, \eta\} \equiv\left\{\sigma_{v}, \eta_{v}\right\}$ for the virus and $\{\sigma, \eta\} \equiv\left\{\sigma_{i s}, \eta_{i s}\right\}$ for the immune system. This landscape is dynamic in that the fitness peak is allowed to shift from one genome to another at specified intervals. To model the interspecies interaction, the master sequence for the immune system is assumed to impose a death rate $\delta$ on the corresponding viral sequence. When this coincides with the viral master sequence, the viral fitness peak shifts out of self-preservation. The new viral master sequence regenerates on a time scale $\tau_{v}$, defined as the time required for the new master sequence to outnumber the old. At this point, the immune fitness peak shifts to match the viral master sequence and will regenerate on a similarly defined timescale $\tau_{i s}$ after which the viral peak shifts again. These steps are iterated so that the virus traverses genome space with the immune system in hot pursuit. Using recent results on the dynamics of a quasispecies on time-dependent landscapes $\stackrel{4}{4}$, Kamp and Bornholdt ${ }^{18,19}$ found expressions for the long-term survival of a conser- 
vatively replicating virus and immune system ${ }^{18.19}$ by considering the behavior of each species on a dynamic fitness landscape. In essence, every time the fitness peak shifts, the concentration of the master sequence drops dramatically and begins to regrow, while the large concentration of the old master sequence drains away. Rigorously, the dynamics of a quasispecies on a single fitness peak landscape follow a set of differential equations defining the evolution of the various genomes in terms of their Hamming distance $H D\left(\phi, \phi^{\prime}\right)$. This is defined as the smallest number of point mutations that lead from $\phi$ to $\phi^{\prime}$. Utilizing the radial symmetry of the fitness landscape, the quasispecies equations become

$$
\begin{aligned}
& \frac{d w_{l, x}}{d t}= \\
& \sum_{l^{\prime}=0}^{l} \frac{\left(n_{x}-l^{\prime}\right) !}{\left(n_{x}-l\right) !} A_{x}\left(l^{\prime}\right)\left(\frac{\epsilon_{x}}{S-1}\right)^{l-l^{\prime}}\left(1-\epsilon_{x}\right)^{n_{x}-\left(l-l^{\prime}\right)} w_{l^{\prime}, x} \\
& -f_{x}(t) w_{l, x},
\end{aligned}
$$

where $x \in\{v, i s\}, w_{l}$ is the concentration of sequences of Hamming distance $l$ from the master sequence, $f_{i s}(t)=$ $\sum_{l} A_{i s}(l) w_{l, i s}=\left(\sigma_{i s}-\eta_{i s}\right) w_{0, i s}+\eta_{i s}$ for the immune system, $f_{v}(t)=\delta$ for the viral sequence that coincides with the immune master sequence and $f_{v}(t)=0$ otherwise. $\epsilon$ represents the point mutation probability (more complex mutations such as insertions and deletions, as well as the possibility of recombination, are ignored).

Building on recent work regarding the dynamics of a quasispecies on mobile fitness landscapes ${ }^{4}$, a number of qualitative features have come to light. Besides the standard (albeit modified by the dynamic nature of the landscape) error catastrophe at high $\epsilon_{v}$, the virus is only viable above a given minimum mutation rate, below which it is unable to keep up with the moving landscape (dubbed the "adaptability" catastrophe). At these low mutation rates, each shift of the landscape is followed by a period of time wherein the new master sequence attempts to build up an equilibrium distribution. However, before the new master sequence can rebuild to the levels reached by the old master before the shift, the fitness peak moves again. As this process repeats, any initial quasispecies will disappear and genomes become stochastically distributed. Further, an optimal mutation rate for the immune system can be found that minimizes the range of viral mutation rates that allow persistence of the viral quasispecies. This optimal rate has been determined and found to be independent of the parameters of the model and the properties of the viral species as well as comparing admirably with the rates of somatic hypermutation in B-cells $\frac{18}{}$. The agreement suggests that this model, although approximate in nature, captures the robust features of the co-evolution of viruses with an adaptive immune system.

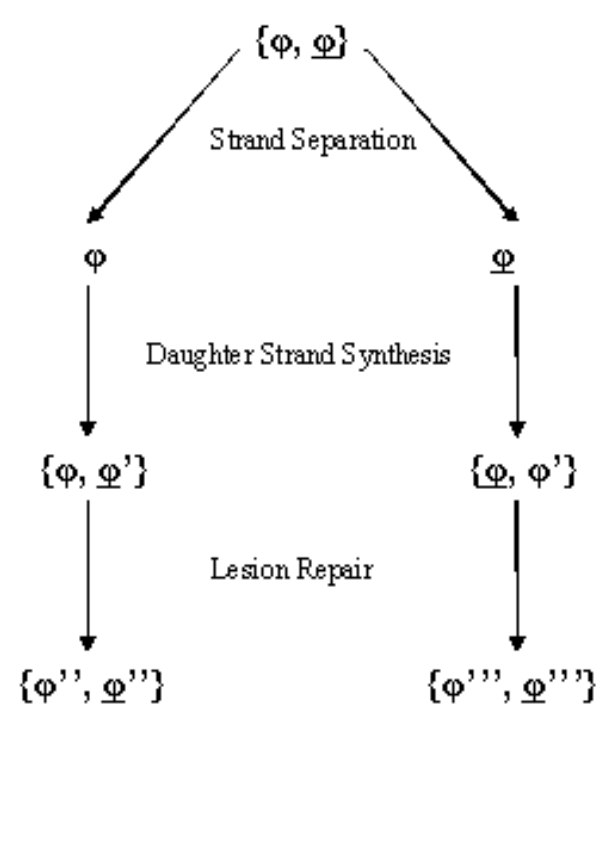

FIG. 1: A schematic model of DNA replication. Adapted from $^{20}$.

Although the host-parasite model has yielded impressive successes, work has been restricted to conservatively replicating systems, which differ greatly from the true semi-conservative systems that dominate nature. In a conservative system, multiple, possibly error prone, copies of an original strand are produced without harming or changing the original strand. Thus, the original quasispecies model is ideally suited for the study of RNA viruses or in-vitro RNA evolution experiments. Semiconservative replication follows a different route shown schematically in Fig. 1. DNA exists as a tightly bound double helix structure, where each strand $\phi$ is connected to a complementary strand $\underline{\phi}$, where $\phi$ represents the complement of strand $\phi$, and can be written as $\underline{s}_{1} \underline{s}_{2} \cdots \underline{s}_{n}$ where we assign the nucleotides $A \equiv 1, G \equiv 2, T \equiv 3, C \stackrel{\underline{s}_{n}}{\equiv}$ 4 and $\underline{s}_{i}=\left(s_{i}+S / 2\right) \bmod S$. In order to undergo replication, the double helix unzips to free two single strands $\phi$ and $\phi$. Each of these is replicated to produce an errorprone complement, yielding $\left\{\phi, \underline{\phi}^{\prime}\right\}$ and $\left\{\underline{\phi}, \phi^{\prime}\right\}$. Each error must result in a base mismatch, which can be recognized and selectively repaired by enzymes in the cell. These enzymes can recognize the new strand and ensure that the mismatch is repaired by replacing the new mismatched base, keeping the effective error rate $\epsilon$ low. In the final stage, the strands become indistinguishable and various maintenance enzymes repair the remaining errors with a 50\% probability of correcting the mismatch in either strand. Thus, the final result are two new pairs, each consisting of two new strands, $\left\{\phi^{\prime \prime}, \phi^{\prime \prime}\right\}$ and $\left\{\phi^{\prime \prime \prime}, \phi^{\prime \prime \prime}\right\}$. Recent work by Tannenbaum et. $a^{2 \overline{20}}$ has extended the 
quasispecies model to the case of semi-conservative replication, which was found to display significantly different behavior in the infinite time limit on a static landscape.

To properly treat a semi-conservative quasispecies model on a single fitness landscape, ignoring back mutations, Eqn. (2) must be recast $a^{20}$

$$
\begin{aligned}
& \frac{d w_{l, x}}{d t}=2 \sum_{l^{\prime}=0}^{l} A_{x}\left(l^{\prime}\right)\left(\frac{\epsilon_{x} / 2}{S-1}\right)^{l-l^{\prime}}\left(1-\frac{\epsilon_{x}}{2}\right)^{n_{x}-l-l^{\prime}} w_{l^{\prime}, x} \\
& -\left(A_{x}(l)+f(t)\right) w_{l, x},
\end{aligned}
$$

where $f(t)$ is defined above. Here, we examine the dynamics of the semi-conservative equations within the confines of Kamp and Bornholdt's model of co-evolution. This study will focus on the dynamical aspects of Eqn. (3), as opposed to the equilibrium effects studied by Tannenbaum et. al $\stackrel{20}{=}$. Following Kamp and Bornholdt, the condition for the viability of the quasispecies is

$$
\kappa_{x} \equiv \frac{w_{1, x}(\tau)}{n_{x} e^{\eta_{x} \tau} w_{0, x}(0)} \geq 1,
$$

where $\kappa$ represents the ratio of master sequence concentrations at the beginning and end of an entire cycle of landscape shifts in an unconstrained system compared to the equivalent growth of a random sequence far from the peak. Obviously, if the master sequence outgrows the random sequence over this period, even with the concentration losses incurred by the peak shift, it will survive for all times. If the master sequence is outgrown by the random sequence, i.e. if $\kappa<1$, the master sequence will diminish and disappear at long times.

After a fair bit of work, the condition for viability of the immune genome can be expressed as ${ }^{21}$

$$
\begin{aligned}
& \kappa_{i s}=\left(\frac{\sigma_{i s} \epsilon_{i s}\left(1-\epsilon_{i s} / 2\right)^{n_{i s}-1}}{(S-1)\left(\sigma_{i s}-\eta_{i s}\right)\left(2\left(1-\epsilon_{i s} / 2\right)^{n_{i s}}-1\right)}\right) \\
& \times\left(e^{\left(2 \sigma_{i s}\left(1-\epsilon_{i s} / 2\right)^{n_{i s}}-\sigma_{i s}-\eta_{i s}\right) \tau}-\right. \\
& \left.e^{\left(2 \eta_{i s}\left(1-\epsilon_{i s} / 2\right)^{n_{i s}}-2 \eta_{i s}\right) \tau}\right) \geq 1 \\
& \tau=\tau_{i s}+\tau_{v} \\
& \tau_{i s}=-\frac{\ln \left(\frac{\left(1-\epsilon_{i s} / 2\right)^{n_{i s}} \epsilon_{i s}}{\left(2\left(1-\epsilon_{i s} / 2\right)^{n_{i s}}-1\right)(S-1)}\right)}{\left.\left(1-\epsilon_{i s} / 2\right)^{n_{i s}}-1\right)\left(\sigma_{i s}-\eta_{i s}\right)}
\end{aligned}
$$

and, for a semi-conservative viral species,

$$
\begin{aligned}
& \kappa_{v}=\left(\frac{\sigma_{v} \epsilon_{v}\left(1-\epsilon_{v} / 2\right)^{n_{v}-1}}{(S-1)\left(\sigma_{v}-\eta_{v}\right)\left(2\left(1-\epsilon_{v} / 2\right)^{n_{v}}-1\right)}\right) \\
& \times\left(e^{\left(2 \sigma_{v}\left(1-\epsilon_{v} / 2\right)^{n_{v}}-\sigma_{v}-\eta_{v}\right) \tau}-\right.
\end{aligned}
$$

$$
\begin{aligned}
& \left.e^{\left(2 \eta_{v}\left(1-\epsilon_{v} / 2\right)^{n_{v}}-2 \eta_{v}\right) \tau}\right) \geq 1 \\
& \tau_{v}=-\frac{\ln \left(\frac{\left(1-\epsilon_{v} / 2\right)^{n_{v}} \epsilon_{v}}{\left(2\left(1-\epsilon_{v} / 2\right)^{n_{v}}-1\right)(S-1)}\right)}{\left(2\left(1-\epsilon_{v} / 2\right)^{n_{v}}-1\right)\left(\sigma_{v}-\eta_{v}\right)+\delta} .
\end{aligned}
$$

A conservatively replicating virus interacting with a semi-conservative immune system will follow the behavior described by the conservative model of Kamp and Bornholdt,

$$
\kappa_{v}=\left(\frac{\left(e^{\left(q_{v}^{n} \sigma_{v}-\eta_{v}\right) \tau}-e^{\left(q_{v}^{n_{v}} \eta_{v}-\eta_{v}\right) \tau}\right)\left(1-q_{v}\right) \sigma_{v}}{(S-1)\left(\sigma_{v}-\eta_{v}\right) q_{v}}\right),
$$

where $q=1-\epsilon$ represents the replicative fidelty per base pair, $\tau=\tau_{i s}+\tau_{v}$ as always, and

$$
\tau_{v}=-\frac{\ln \left(\frac{1-q_{v}}{S-1}\right)}{q_{v}^{n}\left(\sigma_{v}-\eta_{v}\right)+\delta} .
$$

In contrast to previous work, $\tau_{i s}$ is described by the semiconservative result described by Eqn. (7). In Fig. 2 we

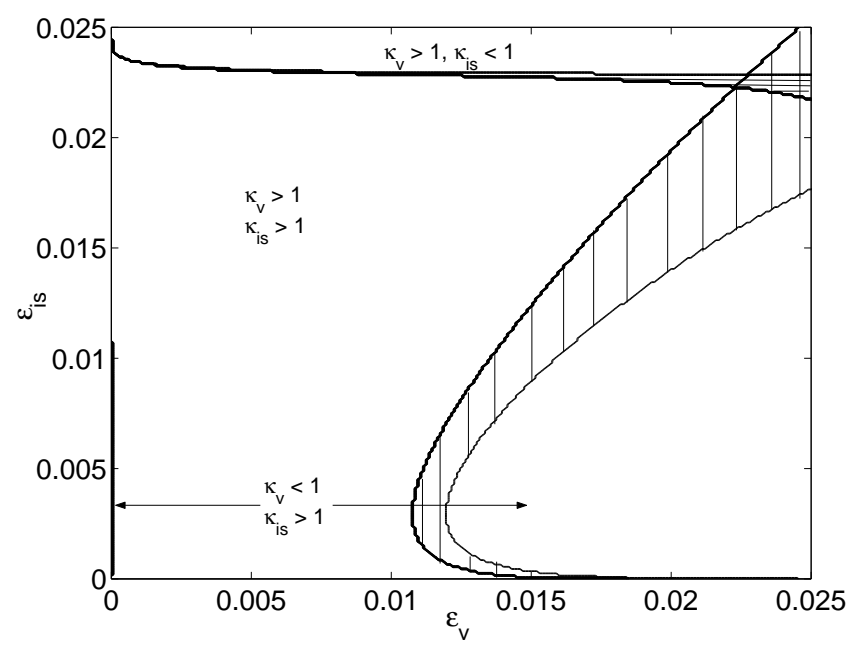

FIG. 2: Regions of viability for a co-evolving host-parasite system. The contours for both semi-conservative and conservative virus interacting with a conservative immune system are shown. The vertical lines represent the region where only the conservative virus is viable, while the horizontal lines represent the region where the immune system is only viable for a conservative virus. $n_{i s}=n_{v}=50, \sigma_{i s}=\sigma_{v}=100, \eta_{i s}=$ $\eta_{v}=1, \delta=200$.

plot the regions of viability defined by $\kappa_{v, i s} \geq 1$ with a particular set of parameters for a semi-conservative immune receptor interacting with both a conservative and semi-conservative virus. It is immediately clear that the conservative virus can survive under a wider range of conditions than the semi-conservative, thus obtaining a selective evolutionary advantage. While the immune system is slightly less robust for the semi-conservative virus, 
this effect is small and lies near the region where the immune system becomes unviable, and hence should have little effect on real systems. Although this behavior is dependent on the parameters of the model, the qualitative trend was robust for the vast majority of biologically reasonable parameter choices. Further, the increased viability lies both near and far from the optimal immune mutation rate, important since a population of viruses in nature is expected to interact with a range of immune system properties, and the behavior away from the optimum should play an important role in the evolution of the system. Thus, we have demonstrated the existence of a clear selective advantage for conservative replication in viral species.

We conclude with a cautionary note. One must al- ways take great care in extracting grand predictions from simplified models such as this one. In nature, a myriad of evolutionary pressures battle for dominance and it is often difficult to pinpoint the selective advantage for a given trait. However, the model presented here likely captures many robust features of the evolution, and suggests a possible explanation for the success of conservative viruses in nature.

\section{Acknowledgments}

The authors gratefully acknowledge useful discussions with Emmanuel Tannenbaum and Brian Dominy.
1 D. Voet and J. Voet, Biochemistry (John Wiley and Sons, Inc., New York, NY, 1995).

2 M. Eigen, Naturwissenschaften 58, 465 (1971).

3 M. Eigen, J. McCaskill, and P. Schuster, Adv. Chem. Phys. 75, 149 (1989).

4 M. Nilsson and N. Snoad, Phys. Rev. Lett. 84, 191 (2000).

5 B. Drossel, Adv. in Phys. 50, 209 (2001).

6 C. O. Wilke, C. Ronnewinkel, and T. Martinetz, Phys. Rep. 349, 395 (2001).

7 P. Tarazona, Phys. Rev. A 45, 6038 (1992).

8 S. Galluccio, Phys. Rev. E 56, 4526 (1997).

9 P. Schuster and P. F. Stadler, Computers Chem. 18, 295 (1994).

10 L. Peliti, cond-mat/9712027 (1997).

11 S. Altmeyer and J. McCaskill, Phys. Rev. Lett. 86, 5819 (2001).

12 P. R. A. Campos and J. F. Fontanari, Phys. Rev. E 58, 2664 (1998).
13 D. Alves and J. F. Fontanari, Phys. Rev. E 57, 7008 (1998).

14 C. O. Wilke, C. Ronnewinkel, and T. Martinetz, Phys. Rep. 349, 395 (2001).

15 J. Krug and C. Karl, Physica A 318, 137 (2003).

16 S. Crotty, C. E. Cameron, and R. Andino, Proc. Nat. Acad. Sci. 98, 6895 (2001).

17 L. A. Loeb, J. M. Essigmann, F. Kazazi, J. Zhang, K. D. Rose, and J. I. Mullins, Proc. Nat. Acad. Sci. 96, 1492 (1999).

18 C. Kamp and S. Bornholdt, Phys. Rev. Lett. 88, 068104 (2002).

19 C. Kamp, C. O. Wilke, C. Adami, and S. Bornholdt, Complexity $\mathbf{8 ,} 28$ (2003).

20 E. Tannenbaum, E. J. Deeds, and E. I. Shakhnovich, Phys. Rev. E (2004), in press.

21 Y. Brumer and E. Shakhnovich (2004), to be published. 